\title{
On uniqueness of recovering the
} convolution integro-differential operator from the spectrum of its non-smooth one-dimensional perturbation

\section{S.A. Buterin ${ }^{*}$ (D) and S.V. Vasiliev}

"Correspondence:

buterinsa@info.sgu.ru

Department of Mathematics,

Saratov University, Saratov, Russia

\section{严 Springer}

\begin{abstract}
We consider a one-dimensional perturbation of the convolution integro-differential operator of arbitrary order on a finite interval. The inverse problem of recovering the convolution component from the spectrum is studied, provided that the perturbation term is known a priori. We prove the uniqueness theorem for this inverse problem and establish the minimal requirements on the perturbation term guarantying the informativity of the spectrum.
\end{abstract}

MSC: $34 \mathrm{~A} 55 ; 45 \mathrm{J05} ; 47 \mathrm{G} 20$

Keywords: Integro-differential operator; One-dimensional perturbation; Convolution; Inverse spectral problem

\section{Introduction}

We study an inverse spectral problem for the integro-differential operator corresponding to the boundary value problem $\mathcal{A}=\mathcal{A}(n, M, g, v)$ of the form

$$
\begin{aligned}
& A y:=i^{n} y^{(n)}+\int_{0}^{x} M(x-t) y^{(n-1)}(t) d t+g(x) \int_{0}^{\pi} y(t) v(t) d t=\lambda y, \quad 0<x<\pi, \\
& y(0)=y^{\prime}(0)=\cdots=y^{(n-1)}(0)=0,
\end{aligned}
$$

where $n \geq 1$ is fixed and $(\pi-x) M(x), g(x), v(x) \in L_{2}(0, \pi)$ are complex-valued functions. Moreover, the functions $g(x)$ and $v(x)$ are supposed to satisfy the following condition:

$$
\int_{a}^{a+\varepsilon}|g(x)| d x>0, \quad \int_{b-\varepsilon}^{b}|v(x)| d x>0
$$

for each positive $\varepsilon$ and with certain fixed $a$ and $b$ obeying the inequality $0 \leq a<b \leq \pi$. Otherwise, the integro-differential operator $A$ is of the Volterra type and, hence, problem (1), (2) becomes an initial value problem with the empty spectrum.

Inverse problems of spectral analysis consist in recovering operators from their spectral characteristics. Such problems often appear in mathematics, mechanics, physics, elec-

(c) The Author(s) 2018. This article is distributed under the terms of the Creative Commons Attribution 4.0 International License (http://creativecommons.org/licenses/by/4.0/), which permits unrestricted use, distribution, and reproduction in any medium, provided you give appropriate credit to the original author(s) and the source, provide a link to the Creative Commons license, and indicate if changes were made. 
tronics, geophysics, meteorology, and other branches of natural sciences and engineering. The greatest success in the inverse problem theory has been achieved for the SturmLiouville operator (see [1-4] and the references therein) and afterwards for higher-order differential operators [5-7]. For integro-differential and other classes of non-local operators, the classical methods of inverse spectral theory either do not work or require essential modifications. Inverse problems for some integro-differential operators were studied in [8-31] and other papers.

Various aspects of recovering the functions $g(x)$ and $v(x)$ from spectral data of the eigenvalue problem $\mathcal{A}$ in the cases $n=1$ and $n=2$, provided that the function $M(x)$ is known a priori and $a=0$, were studied in [9, 17, 20,22, 24]. Moreover, in [19] this inverse problem was studied in the self-adjoint case when $n=2, M(x)=0, g(x)= \pm \overline{v(x)}$ and instead of (2) the Dirichlet boundary conditions $y(0)=y(\pi)=0$ were imposed.

In the present paper we study the following conversed inverse problem.

Inverse problem 1 Given the spectrum $\left\{\lambda_{k}\right\}_{k \geq 1}$ of the problem $\mathcal{A}$, find the function $M(x)$, provided that the functions $g(x)$ and $v(x)$ are known a priori.

A nonlinear inclusion of the function $M(x)$ into the representation of the characteristic function for the boundary value problem $\mathcal{A}$ (see (6) below) makes recovering the function $M(x)$ more difficult than recovering $g(x)$ and $v(x)$. In [13] inverse problem 1 was studied in the case when the functions $g(x), v(x)$ belong to $W_{2}^{1}[0, \pi]$ and satisfy the condition $g(0) v(\pi) \neq 0$. The inverse problem was reduced to a nonlinear integral equation with singularity, whose solvability has been proven in the appropriate class of functions. This allowed to prove the uniqueness theorem and to obtain a constructive procedure for solving the inverse problem along with necessary and sufficient conditions of its solvability. However, in the case $g(x), v(x) \in L_{2}(0, \pi)$, it is not possible to derive such an equation. In the present paper, using another approach, we prove the following uniqueness theorem.

Theorem 1 Assume that the functions $g(x)$ and $v(x)$ are given.

(i) Let $n=1$ or $n=2$. Then the specification of the spectrum $\left\{\lambda_{k}\right\}_{k \geq 1}$ uniquely determines the function $M(x)$ a.e. on $(0, b-a)$.

(ii) Let $n>2$. Then the specification of the spectrum $\left\{\lambda_{k}\right\}_{k \geq 1}$ along with the number $\gamma$ in representation (16) (see below) uniquely determines the function $M(x)$ a.e. on $(0, b-a)$.

(iii) If $g(x)=0$ a.e. on $(0, a)$ and $v(x)=0$ a.e. on $(b, \pi)$, then for any $n \geq 1$ the spectrum $\left\{\lambda_{k}\right\}_{k \geq 1}$ does not depend on behavior of the function $M(x)$ on the interval $(b-a, \pi)$.

Part (iii) of the theorem infers the minimality of the requirements put on $g(x)$ and $v(x)$. In part (i) the number $\gamma$ mentioned in (ii) is determined by the specification of the spectrum. It is valid also for $n>2$ under some additional conditions on $g(x)$ and $v(x)$. For example, in [13] this was established in the case $g(x), v(x) \in W_{2}^{1}[0, \pi]$ when $g(0) v(\pi) \neq 0$. The following uniqueness theorem uses the generalization of this condition.

Theorem 2 Let $n>2$. If $g(x)=0$ a.e. on $(0, a)$ and $v(x)=0$ a.e. on $(b, \pi)$, and additionally the following conditions are satisfied:

$$
\lim _{x \rightarrow a+0} g(x)=d_{a}, \quad \lim _{x \rightarrow b-0} v(x)=d_{b},
$$


where $d_{a} d_{b} \neq 0$, then the specification of the spectrum $\left\{\lambda_{k}\right\}_{k \geq 1}$ uniquely determines the function $M(x)$ a.e. on $(0, b-a)$, provided that the functions $g(x)$ and $v(x)$ are known a priori.

We also note that in the case of $n=1,2, g(x) \equiv 0$ and appropriate boundary conditions, various aspects of recovering the function $M(x)$ from the spectrum were studied in [8, 9, $11,12,14,16,18,21,22,24-29]$ and other works. In [30] and [31] the case of fractional order $n \notin \mathbb{N}$ was studied (see also [32,33] for fractional integro-differential operators and singular integral operators).

The paper is organized as follows. In the next section we introduce and investigate the characteristic function $\Delta(\lambda)$ of the boundary value problem $\mathcal{A}$. In Sect. 3 we study some aspects of recovering $\Delta(\lambda)$ from its zeros. Theorems 1 and 2 are proven in Sect. 4.

\section{Preliminary information}

Let $y=g_{n}(x, \lambda), x \in[0, \pi]$, be a solution of the Cauchy problem

$$
\ell_{n} y:=i^{n} y^{(n)}+\int_{0}^{x} M(x-t) y^{(n-1)}(t) d t+g(x)=\lambda y, \quad y^{(j)}(0)=0, \quad j=\overline{0, n-1} .
$$

Consider the function

$$
\Delta(\lambda)=1-\int_{0}^{\pi} v(x) g_{n}(x, \lambda) d t
$$

which is called the characteristic function of the boundary value problem $\mathcal{A}$.

Lemma 1 Eigenvalues of the problem $\mathcal{A}$ coincide with zeros of its characteristic function.

Proof Let $\Delta\left(\lambda_{0}\right)=0$. Then the function $g_{n}\left(x, \lambda_{0}\right)$ is, obviously, a solution of the boundary value problem $\mathcal{A}$. Since $g(x) \not \equiv 0$, we have $g_{n}(x, \lambda) \not \equiv 0$ for each $\lambda$ and, hence, $g_{n}\left(x, \lambda_{0}\right)$ is an eigenfunction, while $\lambda_{0}$ is the corresponding eigenvalue.

Conversely, if $\lambda_{0}$ is an eigenvalue of $\mathcal{A}$ and $y_{0}(x)$ is a related eigenfunction, then

$$
\int_{0}^{\pi} y_{0}(x) v(x) d x \neq 0
$$

Indeed, otherwise $y_{0}(x)$ would be identically zero as a solution of the homogenous Cauchy problem. Thus, without loss of generality, we can assume that the integral in (7) equals 1. Then we have $y_{0}(x)=g_{n}\left(x, \lambda_{0}\right)$ and, hence, $\Delta\left(\lambda_{0}\right)=0$.

Lemma 2 The solution $y=g_{n}(x, \lambda)$ of the Cauchy problem (5) has the form

$$
g_{n}(x, \lambda)=-\int_{0}^{x} S_{n}(x-t, \lambda) g(t) d t \quad 0<x<\pi,
$$

where the function $y=S_{n}(x, \lambda)$ is a solution of the following Cauchy problem:

$$
\begin{aligned}
& i^{n} y^{(n)}+\int_{0}^{x} M(x-t) y^{(n-1)}(t) d t=\lambda y, \quad 0<x<\pi, \\
& y^{(j)}(0)=\delta_{j, n-1}(-i)^{n}, \quad j=\overline{0, n-1} .
\end{aligned}
$$

Here, $\delta_{j, n-1}$ is the Kronecker delta. 
Proof Consider the Volterra integro-differential operator $L_{\lambda}$ of the form

$$
L_{\lambda} y:=i^{n} y^{(n)}+\int_{0}^{x} M(x-t) y^{(n-1)}(t) d t-\lambda y, \quad 0<x<\pi, \quad y^{(j)}(0)=0, \quad j=\overline{0, n-1} .
$$

By substitution it is easy to check that the inverse operator $M_{\lambda}:=L_{\lambda}^{-1}$ has the form

$$
M_{\lambda} f(x)=\int_{0}^{x} S_{n}(x-t, \lambda) f(t) d t, \quad 0<x<\pi
$$

where $S_{n}(x, \lambda)$ is the solution of (9). Since $g_{n}(x, \lambda)=-M_{\lambda} g$, we arrive at (8).

Put

$$
\begin{aligned}
& S_{n, 0}(x, \lambda)=\frac{1}{i n \rho^{n-1}} \sum_{j=1}^{n} \omega_{j} \exp \left(-i \omega_{j} \rho x\right), \\
& \rho^{n}=\lambda, \quad \omega_{j}=\exp \left(\frac{2 \pi i(j-1)}{n}\right), \quad j=\overline{1, n},
\end{aligned}
$$

i.e., $\omega_{j}$ are all $n$th roots of unity. By virtue of Theorem 2.2 in [34], for any function $M(x)$, $(\pi-x) M(x) \in L_{2}(0, \pi)$, the integral equation

$$
M(x)=n i^{n-1} M_{1}(x)+\sum_{j=2}^{n}\left(\begin{array}{l}
n \\
j
\end{array}\right) i^{n-j} \int_{0}^{x} \frac{(x-t)^{j-2}}{(j-2) !} M_{1}^{* j}(t) d t, \quad 0<x<\pi,
$$

has a unique solution $M_{1}(x),(\pi-x) M_{1}(x) \in L_{2}(0, \pi)$, where

$$
\left(\begin{array}{l}
n \\
j
\end{array}\right)=\frac{n !}{j !(n-j) !}, \quad M_{1}^{* 1}(x)=M_{1}(x), \quad M_{1}^{*(j+1)}(x)=\int_{0}^{x} M_{1}(x-t) M_{1}^{* j}(t) d t, \quad j \geq 1 .
$$

The following lemma is a generalization of Lemma 2.1 in [14].

Lemma 3 The solution $S_{n}(x, \lambda)$ of the Cauchy problem (9) admits the following representation:

$$
S_{n}(x, \lambda)=S_{n, 0}(x, \lambda)+\int_{0}^{x} P(x, t) S_{n, 0}(x-t, \lambda) d t
$$

where

$$
P(x, t)=\sum_{\nu=1}^{\infty} i^{\nu} \frac{(x-t)^{\nu}}{\nu !} M_{1}^{* \nu}(t)
$$

Proof We note that in [14] representation (12) was established for $n=1,2$. Let us generalize its proof for an arbitrary $n$. Consider the integro-differential operators

$$
J_{n} y:=i^{n} y^{(n)}+\int_{0}^{x} M(x-t) y^{(n-1)}(t) d t, \quad J_{1} y:=i y^{\prime}+\int_{0}^{x} M_{1}(x-t) y(t) d t
$$


where $M_{1}(x)$ is the solution of nonlinear integral equation (11). It is easy to show by induction that $J_{n} y=J_{1}^{n} y$, where $J_{1}^{1} y=J_{1} y, J_{1}^{k+1} y=J_{1}\left(J_{1}^{k} y\right), k \geq 1$, for any sufficiently smooth function $y(x)$ obeying the conditions $y^{(k)}(0)=0, k=\overline{0, n-2}$. Consider the right-hand side of (12):

$$
y(x):=S_{n, 0}(x, \lambda)+\int_{0}^{x} P(x, t) S_{n, 0}(x-t, \lambda) d t
$$

Since $S_{n, 0}^{(k)}(0, \lambda)=\delta_{k, n-1}(-i)^{n}, k=\overline{0, n-1}$, we get

$$
y^{(k)}(x)=S_{n, 0}^{(k)}(x, \lambda)+\sum_{j=0}^{k}\left(\begin{array}{l}
k \\
j
\end{array}\right) \int_{0}^{x} \frac{\partial^{k}}{\partial x^{k}} P(x, t) S_{n, 0}^{(k-j)}(x-t, \lambda) d t, \quad k=\overline{0, n-1},
$$

and, hence, $y^{(k)}(0)=\delta_{k, n-1}(-i)^{n}, k=\overline{0, n-1}$. Thus, the right-hand side of (12) satisfies the equation $J_{n} y=J_{1}^{n} y$. Since representation (12) is valid for $n=1$, we have

$$
y(x)=\frac{1}{n \rho^{n-1}} \sum_{j=1}^{n} \omega_{j} S_{1}\left(x, \omega_{j} \rho\right) .
$$

Consequently, we have

$$
J_{n} y=J_{1}^{n} y=\frac{1}{n \rho^{n-1}} \sum_{j=1}^{n} \omega_{j} J_{1}^{n} S_{1}\left(x, \omega_{j} \rho\right)=\frac{\rho}{n} \sum_{j=1}^{n} \omega_{j}^{n+1} S_{1}\left(x, \omega_{j} \rho\right)=\rho^{n} y=\lambda y .
$$

Thus, the right-hand side of (12) satisfies the same Cauchy problem (9) as the function $S_{n}(x, \lambda)$ does and, hence, representation (12) holds.

The following lemma gives another representation for the characteristic function.

Lemma 4 The following representation holds:

$$
\Delta(\lambda)=1+\int_{0}^{\pi} \mu(x) S_{n, 0}(x, \lambda) d x
$$

where

$$
\mu(x)=\mu_{0}(x)+\int_{x}^{\pi} P(t, t-x) \mu_{0}(t) d t, \quad \mu_{0}(x)=\int_{x}^{\pi} v(t) g(t-x) d t .
$$

Proof Substituting (8) into (6) and changing the order of integration, we obtain

$$
\Delta(\lambda)=1+\int_{0}^{\pi} \mu_{0}(x) S_{n}(x, \lambda) d x .
$$

Further, substituting (12) into this formula and changing the order of integration, we arrive at (14) and (15). 


\section{On determination of $\Delta(\lambda)$ by the spectrum}

In what follows, along with $\mathcal{A}$ we consider the boundary value problem $\tilde{\mathcal{A}}=\mathcal{A}(n, \tilde{M}, v, g)$ of the same order $n$ and with the same functions $g(x)$ and $v(x)$. We agree that if a certain symbol $\alpha$ denotes an object related to $\mathcal{A}$, then this object with tilde $\tilde{\alpha}$ denotes the analogous object corresponding to $\tilde{\mathcal{A}}$, and $\hat{\alpha}=\alpha-\tilde{\alpha}$.

Since the characteristic function is an entire function of order $1 / n$, according to the Hadamard factorization theorem, it has the following representation:

$$
\Delta(\lambda)=\gamma \exp \left(\delta_{1, n} \beta \lambda\right) \lambda^{s} \prod_{\lambda_{k} \neq 0}\left(1-\frac{\lambda}{\lambda_{k}}\right) \exp \left(\delta_{1, n} \frac{\lambda}{\lambda_{k}}\right)
$$

where $s$ is the algebraic multiplicity of the eigenvalue $\lambda=0$, while $\gamma \neq 0$ and $\beta$ are some constants.

Lemma 5 Let $n \in\{1,2\}$. If $\left\{\lambda_{k}\right\}_{k \geq 1}=\left\{\tilde{\lambda}_{k}\right\}_{k \geq 1}$, then $\Delta(\lambda) \equiv \tilde{\Delta}(\lambda)$. In other words, the specification of the spectrum uniquely determines the characteristic function.

Proof Taking (16) into account, it remains to prove that the specification of the spectrum uniquely determines $\gamma$ and $\beta$. By virtue of (10) and (14), we have $\Delta(\lambda) \rightarrow 1$ as $\lambda \rightarrow+\infty$. Hence, the coincidence of the spectra $\left\{\lambda_{k}\right\}_{k \geq 1}$ and $\left\{\tilde{\lambda}_{k}\right\}_{k \geq 1}$ implies

$$
\frac{\gamma}{\tilde{\gamma}} \exp \left(\delta_{1, n}(\beta-\tilde{\beta}) \lambda\right)=\frac{\Delta(\lambda)}{\tilde{\Delta}(\lambda)} \rightarrow 1, \quad \lambda \rightarrow+\infty
$$

which is possible only if $\gamma=\tilde{\gamma}$ and $\beta=\tilde{\beta}$.

Lemma 6 Let $n>2$. If the functions $g(x)$ and $v(x)$ satisfy the hypothesis of Theorem 2, then the specification of the spectrum uniquely determines $\gamma$ in representation (16).

Proof Using (13) and (15), it is easy to check that $\mu(x)=0$ on $[b-a, \pi]$. Moreover, from (4) it follows that

$$
\eta(x):=\mu(b-a-x)=d_{a} d_{b} x(1+o(1)), \quad x \rightarrow+0 .
$$

Denote

$$
\delta_{1}(\rho):=\frac{1}{i n \rho^{n-1}} \int_{0}^{b-a} \mu(x) \exp (-i \rho x) d x, \quad \delta_{2}(\rho):=\Delta\left(\rho^{n}\right)-\delta_{1}(\rho)
$$

From (17) it follows that

$$
\delta_{1}(i t) \sim \frac{d_{a} d_{b}}{i^{n} n t^{n+1}} \exp ((b-a) t), \quad t \rightarrow+\infty .
$$

Indeed, we have

$$
\int_{0}^{b-a} \mu(x) \exp (-i \rho x) d x=\exp (-i \rho(b-a)) \int_{0}^{b-a} \eta(x) \exp (i \rho x) d x
$$


Substituting $\rho=i t$ into the last integral and using (17), we get

$$
\begin{aligned}
& \int_{0}^{b-a} \eta(x) \exp (-t x) d x \\
& \quad=\int_{0}^{\varepsilon} \eta(x) \exp (-t x) d x+\int_{\varepsilon}^{b-a} \eta(x) \exp (-t x) d x \\
& \quad=d_{a} d_{b} \int_{0}^{\varepsilon} x \exp (-t x) d x\left(1+h_{\varepsilon}\right)+O(\exp (-\varepsilon t)), \quad \varepsilon \in(0, b-a), t \rightarrow+\infty,
\end{aligned}
$$

where $h_{\varepsilon}=o(1)$ as $\varepsilon \rightarrow 0$. Integrating by parts, we calculate

$$
\int_{0}^{\varepsilon} x \exp (-t x) d x=\frac{1}{t^{2}}-\frac{1}{t^{2}} \exp (-\varepsilon t)-\frac{\varepsilon}{t} \exp (-\varepsilon t) .
$$

Hence,

$$
\int_{0}^{b-a} \eta(x) \exp (-t x) d x \sim \frac{d_{a} d_{b}}{t^{2}}, \quad t \rightarrow+\infty
$$

which along with (18) and (20) gives (19).

Further, by virtue of (10), (14), and (18) and our assumptions on $g(x)$ and $v(x)$, we get

$$
\begin{aligned}
\left|\delta_{2}(i t)\right| & <1+\frac{1}{t^{n-1}} \int_{0}^{b-a}|\mu(x)| \exp \left(t x \operatorname{Re}\left(\omega_{2}\right)\right) d x \\
& =O\left(\exp \left((b-a) t \cos \frac{2 \pi}{n}\right)\right), \quad t \rightarrow+\infty
\end{aligned}
$$

which along with (18) and (19) gives

$$
\Delta\left((i t)^{n}\right) \sim \frac{d_{a} d_{b}}{i^{n} n t^{n+1}} \exp ((b-a) t), \quad t \rightarrow+\infty .
$$

Thus, if $\left\{\lambda_{k}\right\}_{k \geq 1}=\left\{\tilde{\lambda}_{k}\right\}_{k \geq 1}$, then (16) implies

$$
\frac{\gamma}{\tilde{\gamma}}=\frac{\Delta(i t)}{\tilde{\Delta}(i t)} \rightarrow 1, \quad t \rightarrow+\infty,
$$

and, hence, $\gamma=\tilde{\gamma}$.

Remark 1 Lemma 6 implies that, under the hypothesis of Theorem 2, the specification of the spectrum uniquely determines the characteristic function also in the case $n>2$.

\section{Proof of Theorem 1 and Theorem 2}

Along with the problem $\mathcal{A}=\mathcal{A}(1, M, g, v)$, we consider the problem $\mathcal{A}^{*}=\mathcal{A}(1, M, u, w)$, where $w(x)=g(\pi-x)$ and $u(x)=v(\pi-x)$. Let $y=u_{1}(x, \lambda)$ be the solution of the Cauchy problem

$$
\ell_{1}^{*} y:=i y^{\prime}+\int_{0}^{x} M(x-t) y(t) d t+u(x)=\lambda y, \quad 0<x<\pi, y(0)=0 .
$$


According to Lemma 2, the following representation holds:

$$
u_{1}(x, \lambda)=-\int_{0}^{x} S_{1}(x-t, \lambda) u(t) d t
$$

Lemma 7 Let $n=1$. Then the following relation holds:

$$
\hat{\Delta}(\lambda)=-\int_{0}^{\pi} N(x) \mathcal{M}(x, \lambda) d x,
$$

where

$$
\begin{aligned}
& N(x)=\int_{x}^{\pi} \hat{M}(\pi-t) d t \int_{0}^{t-x} u(\tau) g(t-x-\tau) d \tau, \\
& \mathcal{M}(x, \lambda)=\int_{0}^{x} S_{1}(x-t, \lambda) \tilde{S}_{1}(t, \lambda) d t .
\end{aligned}
$$

Proof According to (5) and (21), we have

$$
\begin{aligned}
0= & u_{1}(\pi-x, \lambda) \tilde{\ell}_{1} \tilde{g}_{1}(x, \lambda)-\tilde{g}_{1}(x, \lambda) \ell_{1}^{*} u_{1}(\pi-x, \lambda) \\
= & i\left(u_{1}(\pi-x, \lambda) \tilde{g}_{1}(x, \lambda)\right)^{\prime} \\
& +u_{1}(\pi-x, \lambda)\left(\int_{0}^{x} \tilde{M}(x-t) \tilde{g}_{1}(t, \lambda) d t+g(x)\right) \\
& -\tilde{g}_{1}(x, \lambda)\left(\int_{0}^{\pi-x} \tilde{M}(\pi-x-t) u_{1}(t, \lambda) d t+v(x)\right) .
\end{aligned}
$$

Integrating this from 0 to $\pi$ and changing the order of integration, we arrive at

$$
\Delta^{*}(\lambda)-\tilde{\Delta}(\lambda)=-\int_{0}^{\pi} \hat{M}(\pi-x) d x \int_{0}^{x} u_{1}(t, \lambda) \tilde{g}_{1}(x-t, \lambda) d t,
$$

where

$$
\Delta^{*}(\lambda)=1-\int_{0}^{\pi} w(x) u_{1}(x, \lambda) d x
$$

Formula (25), in particular, gives $\Delta^{*}(\lambda)=\Delta(\lambda)$ and, hence,

$$
\hat{\Delta}(\lambda)=-\int_{0}^{\pi} \hat{M}(\pi-x) d x \int_{0}^{x} u_{1}(t, \lambda) \tilde{g}_{1}(x-t, \lambda) d t .
$$

Substituting (8) for $n=1$ and (22) into (26) and changing the order of integration, we arrive at (23) and (24).

Lemma 8 The following representation holds:

$$
\mathcal{M}(x, \lambda)=-\left(x \exp (-i \lambda x)+\int_{0}^{x} K(x, t) \exp (-i \lambda t) d t\right), \quad 0 \leq x \leq \pi,
$$

where $|K(x, t)| \leq f(x-t)$ with some $f(x) \in L_{2}(0, \pi)$. 
Proof Substituting (12) for $n=1$ into the second formula in (24), we get

$$
\mathcal{M}_{1}(x, \lambda)=-\left(x \exp (-i \lambda x)+\sum_{j=1}^{3} \mathcal{M}_{j}(x, \lambda)\right)
$$

where, putting $Q(x, t)=P(x, x-t)$, we have

$$
\begin{aligned}
& \mathcal{M}_{1}(x, \lambda)=\int_{0}^{x} \exp (-i \lambda t) d t \int_{0}^{x-t} Q(x-t, \tau) \exp (-i \lambda \tau) d \tau \\
& \mathcal{M}_{2}(x, \lambda)=\int_{0}^{x} \exp (-i \lambda t) d t \int_{0}^{x-t} \tilde{Q}(x-t, \tau) \exp (-i \lambda \tau) d \tau \\
& \mathcal{M}_{3}(x, \lambda)=\int_{0}^{x} d t \int_{0}^{t} Q(t, \tau) \exp (-i \lambda \tau) d \tau \int_{0}^{x-t} \tilde{Q}(x-t, \xi) \exp (-i \lambda \xi) d \xi
\end{aligned}
$$

Combining the exponentials and changing the order of integration in the last three formulae, we arrive at (27) with

$$
\begin{aligned}
K(x, t)= & \int_{0}^{t} Q(x-\tau, t-\tau) d \tau+\int_{0}^{t} \tilde{Q}(x-\tau, t-\tau) d \tau \\
& +\int_{0}^{t} d \tau \int_{\tau}^{x+\tau-t} Q(\xi, \tau) \tilde{Q}(x-\xi, t-\tau) d \xi,
\end{aligned}
$$

which along with (13) finishes the proof.

Now we are in a position to give the proof of Theorem 1. First, let $n=1$. Changing the order of integration in (24), we get

$$
N(x)=\int_{x}^{\pi} v(t) d t \int_{0}^{t-x} \hat{M}(t-x-\tau) g(\tau) d \tau .
$$

Without loss of generality, we assume that $g(x)=0$ a.e. on $(0, a)$ and $v(x)=0$ a.e. on $(b, \pi)$. Otherwise, the points $a>0$ and $b<\pi$ could be shifted closer to the points 0 and $\pi$, respectively, which would make Theorem 1 even stronger. Denote

$$
g_{1}(x):=g(x+a), \quad x \in(0, \pi-a), \quad v_{1}(x):=v(b-x), \quad x \in(0, b) .
$$

Then, by virtue of (3), we get

$$
\int_{0}^{\varepsilon}\left|g_{1}(x)\right| d x>0, \quad \int_{0}^{\varepsilon}\left|v_{1}(x)\right| d x>0, \quad \varepsilon>0 .
$$

According to (28), we have $N(x)=0$ on $(b, \pi)$ and

$$
\begin{aligned}
N(b-x) & =\int_{0}^{x} \nu_{1}(t) d t \int_{0}^{x-t} \hat{M}(x-t-\tau) g(\tau) d \tau \\
& =\int_{0}^{x} g(t) d t \int_{0}^{x-t} \hat{M}(x-t-\tau) \nu_{1}(\tau) d \tau
\end{aligned}
$$


for $x \in(0, b)$. Hence, $N(b-x)=0$ for $x \in(0, a)$, i.e., $N(x)=0$ on $(b-a, \pi)$, and

$$
\begin{aligned}
& N(b-a-x)=\int_{0}^{x} g_{1}(t) F(x-t) d t \\
& F(x)=\int_{0}^{x} \hat{M}(x-t) v_{1}(t) d t, \quad x \in(0, b-a) .
\end{aligned}
$$

Further, substituting (27) into (23) and changing the order of integration, we obtain

$$
\hat{\Delta}(\lambda)=\int_{0}^{\pi}\left(x N(x)+\int_{x}^{\pi} K(t, x) N(t) d t\right) \exp (-i \lambda x) d x
$$

According to Lemma 5, the coincidence of the spectra gives

$$
x N(x)+\int_{x}^{\pi} K(t, x) N(t) d t=0 \quad \text { a.e. on }(0, \pi)
$$

which implies that $N(x)=0$ a.e. on $(0, \pi)$. By virtue of the first inequality in (29) and (30) along with the Titchmarsh theorem (see [35]), we obtain $F(x)=0$ a.e. on $(0, b-a)$. Applying the Titchmarsh theorem to the second equality in (30) and taking into account the second inequality in (29), we arrive at $\hat{M}(x)=0$ a.e. on $(0, b-a)$. Thus, part (i) of Theorem 1 is proven for $n=1$.

Let $n>1$. Along with $\mathcal{A}$ we consider the problem $\mathcal{A}_{1}=\mathcal{A}\left(1, M_{1}, g\right.$,v), where the function $M_{1}(x)$ is connected with $M(x)$ by relation (11). According to Lemma 4 , the characteristic function $\Delta_{1}(\lambda)$ of $\mathcal{A}_{1}$ has the form

$$
\Delta_{1}(\lambda)=1-i \int_{0}^{\pi} \mu(x) \exp (-i \lambda x) d x
$$

with the same function $\mu(x)$ that appears in representation (14) for the characteristic function $\Delta(\lambda)$ of $\mathcal{A}$.

Further, if $\gamma=\tilde{\gamma}$ and $\left\{\lambda_{k}\right\}_{k \geq 1}=\left\{\tilde{\lambda}_{k}\right\}_{k \geq 1}$, then formula (16) implies $\Delta(\lambda) \equiv \tilde{\Delta}(\lambda)$. By virtue of (14), this infers $\mu(x)=\tilde{\mu}(x)$ and, hence, (31) gives $\Delta_{1}(\lambda) \equiv \tilde{\Delta}_{1}(\lambda)$. According to part (i) of Theorem 1 for $n=1$, we get $M_{1}(x)=\tilde{M}_{1}(x)$ a.e. on $(0, b-a)$ and, by virtue of (11), $M(x)=\tilde{M}(x)$ a.e. on $(0, b-a)$. Thus, part (i) for $n=2$ and part (ii) of Theorem 1 are proven. We actually have established that $\Delta(\lambda) \equiv \tilde{\Delta}(\lambda)$ if and only if $\hat{M}(x)=0$ a.e. on $(0, b-a)$, which proves part (iii) of Theorem 1 .

For the proof of Theorem 2, it remains to note that under its hypothesis, according to Lemma 6 , the specification of the spectrum uniquely determines $\gamma$.

\section{Conclusions}

We have obtained uniqueness results for the inverse problem of recovering the $n$th order convolution integro-differential operator from the spectrum of its one-dimensional perturbation, when the functions $g(x)$ and $v(x)$, involved into the perturbation term, belong to $L_{2}(0, \pi)$. The most minimal requirements for the functions $g(x)$ and $v(x)$ were established guaranteeing the uniqueness in the case $n=1,2$. The case $n>2$ is more complicated and for the uniqueness theorem to hold it requires some additional assumption about $g(x)$ and 
$v(x)$. As such an assumption we have used condition (4). However, there exist other possible assumptions that would guarantee the uniqueness. For example, one could use the following generalization of (4):

$$
g(x) \sim d_{a} x^{\alpha}, \quad x \rightarrow a+0, \quad v(x) \sim d_{b} x^{\beta}, \quad x \rightarrow b-0,
$$

for some fixed $\alpha, \beta>-1 / 2$ and $d_{a} d_{b} \neq 0$. We note that earlier this inverse problem was studied only in the case $g(x), v(x) \in W_{2}^{1}[0, \pi], g(0) v(\pi) \neq 0$.

Studying inverse problems for one-dimensional perturbations of integro-differential and integral operators is a prospective direction in the inverse spectral theory because many important operators can be represented in this form. For example, in [36] it was shown how the uniqueness theorem for the classical inverse Sturm-Liouville problem follows from a uniqueness theorem for the one-dimensional perturbation of a Volterra integral operator.

\section{Acknowledgements}

This work was supported by Grant 17-11-01193 of the Russian Science Foundation.

\section{Abbreviations \\ Not applicable.}

Availability of data and materials

Not applicable.

Ethics approval and consent to participate

Not applicable.

\section{Competing interests}

The authors declare that they have no competing interests.

Consent for publication

Not applicable.

Authors' contributions

All authors contributed equally and read and approved the final version of the manuscript.

\section{Publisher's Note}

Springer Nature remains neutral with regard to jurisdictional claims in published maps and institutional affiliations.

Received: 24 October 2017 Accepted: 5 April 2018 Published online: 13 April 2018

\section{References}

1. Borg, G.: Eine umkehrung der Sturm-Liouvilleschen eigenwertaufgabe. Acta Math. 78, 1-96 (1946)

2. Marchenko, V.A.: Sturm-Liouville Operators and Their Applications. Naukova Dumka, Kiev (1977); English transl., Birkhäuser, 1986

3. Levitan, B.M.: Inverse Sturm-Liouville Problems. Nauka, Moscow (1984); English transl., VNU Sci. Press, Utrecht, 1987

4. Freiling, G., Yurko, V.A.: Inverse Sturm-Liouville Problems and Their Applications. Nova Publ. (Nova Science Publishers), New York (2001)

5. Beals, R., Deift, P., Tomei, C.: Direct and Inverse Scattering on the Line. Mathematica Surveys and Monographs, vol. 28. AMS, Providence (1988)

6. Yurko, V.A.: Inverse Spectral Problems for Differential Operators and Their Applications. Gordon \& Breach, Amsterdam (2000)

7. Yurko, V.A.: Method of Spectral Mappings in the Inverse Problem Theory. Inverse and III-Posed Problems Series. VSP, Utrecht (2002)

8. Malamud, M.M.: On some inverse problems. In: Boundary Value Problems of Mathematical Physics, Kiev, pp. 116-124 (1979) (in Russian)

9. Yurko, V.A.: Inverse problem for integro-differential operators of the first order. In: Funkt. Analiz, Ul'yanovsk, vol. 22, pp. 144-151 (1984) (in Russian)

10. Eremin, M.S.: An inverse problem for a second-order integro-differential equation with a singularity. Differ. Uravn. 24(2), 350-351 (1988)

11. Yurko, V.A.: An inverse problem for integro-differential operators. Mat. Zametki 50(5), 134-146 (1991) (in Russian); English transl. in Math. Notes 50(5-6), 1188-1197 (1991) 
12. Buterin, S.A.: Recovering a convolution integro-differential operator from the spectrum. In: Matematika. Mekhanika, vol. 6, pp. 15-18. Saratov University, Saratov (2004) (in Russian)

13. Buterin, S.A.: On an inverse problem for non-Volterra integro-differential equations. In: Matematika. Mekhanika, vol. 8, pp. 22-24. Saratov University, Saratov (2006) (in Russian)

14. Buterin, S.A.: On an inverse spectral problem for a convolution integro-differential operator. Results Math. 50(3-4), 173-181 (2007)

15. Kuryshova, JV.: Inverse spectral problem for integro-differential operators. Mat. Zametki 81(6), 855-866 (2007) (in Russian); English transl. in Math. Notes 81(6), 767-777 (2007)

16. Buterin, S.A.: On the reconstruction of a convolution perturbation of the Sturm-Liouville operator from the spectrum. Differ. Uravn. 46, 146-149 (2010) (in Russian); English transl. in Differ. Equ. 46, 150-154 (2010)

17. Kuryshova, Y: An inverse spectral problem for differential operators with integral delay. Tamkang J. Math. 42(3), 295-303 (2011)

18. Wang, Y., Wei, G.: The uniqueness for Sturm-Liouville problems with aftereffect. Acta Math. Sci. 32A(6), 1171-1178 (2012)

19. Zolotarev, V.A.: An inverse problem for the Sturm-Liouville operator with non-local potential. Rep. Natl. Acad. Sci. Ukr. 8,7-12 (2012) (in Russian)

20. Yurko, V.A.: An inverse spectral problem for integro-differential operators. Far East J. Math. Sci. 92(2), 247-261 (2014)

21. Buterin, S.A., Choque Rivero, A.E.: On inverse problem for a convolution integro-differential operator with Robin boundary conditions. Appl. Math. Lett. 48, 150-155 (2015)

22. Yurko, V.A.: Inverse problems for integro-differential operators of the first order. Mat. Zametki 100(6), 946-953 (2016) (in Russian); English transl. in Math. Notes 100(6), 876-882 (2016)

23. Yurko, V.A.: Inverse problems for second order integro-differential operators. Appl. Math. Lett. 74, 1-6 (2017)

24. Yurko, V.A.: Inverse spectral problems for first order integro-differential operators. Bound. Value Probl. 2017, 98 (2017) https://doi.org/10.1186/s13661-017-0831-8

25. Bondarenko, N., Buterin, S.: On recovering the Dirac operator with an integral delay from the spectrum. Results Math. 71(3-4), 1521-1529 (2017)

26. Bondarenko, N.P.: Inverse problem for the Dirac system with an integral delay of the convolution-type. In: Matematika. Mekhanika, vol. 19, pp. 9-12. Saratov University, Saratov (2017)

27. Buterin, S.A., Sat, M.: On the half inverse spectral problem for an integro-differential operator. Inverse Probl. Sci. Eng 25(10), 1508-1518 (2017)

28. Buterin, S.A.: On inverse spectral problems for first-order integro-differential operators with discontinuities. Appl. Math. Lett. 78, 65-71 (2018)

29. Bondarenko, N.P.: An inverse problem for an integro-differential operator on a star-shaped graph. Math. Methods Appl. Sci. 41(4), 1697-1702 (2018)

30. Ignatyev, M.: On an inverse spectral problem for the convolution integro-differential operator of fractional order. Results Math. 73, 34 (2018) https://doi.org/10.1007/s00025-018-0800-2

31. Ignatiev, M.: On an inverse spectral problem for one integro-differential operator of fractional order. J. Inverse III-Posed Probl., (2018). https://doi.org/10.1515/jiip-2017-0121

32. Denton, Z., Ramírez, J.D.: Existence of minimal and maximal solutions to RL fractional integro-differential initial value problems. Opusc. Math. 37(5), 705-724 (2017)

33. Xiao, J: A new perspective on the Riesz potential. Adv. Nonlinear Anal. 6(3), 317-326 (2017)

34. Buterin, S.A.: Inverse spectral reconstruction problem for the convolution operator perturbed by a one-dimensional operator. Mat. Zametki 80(5), 668-682 (2006) (Russian); English transl. in Math. Notes 80(5), 631-644 (2006)

35. Titchmarsh, E.C.: Theory of Functions. Oxford University Press, Oxford (1939)

36. Yurko, V.A.: An inverse problem for integral operators. Mat. Zametki 37, 690-701 (1985); English transl. in Math. Notes 37, 378-385 (1985)

\section{Submit your manuscript to a SpringerOpen ${ }^{\circ}$ journal and benefit from:}

- Convenient online submission

- Rigorous peer review

- Open access: articles freely available online

- High visibility within the field

- Retaining the copyright to your article

Submit your next manuscript at $\gg$ springeropen.com 